\title{
Shoulder Long Head Biceps Tendon Pathology Is Associated With Increasing Rotator Cuff Tear Size
}

\author{
Vittorio Candela, M.D., Jacopo Preziosi Standoli, P.T., Stefano Carbone, M.D., Ph.D., \\ Marco Rionero, M.D., and Stefano Gumina, M.D., Ph.D.
}

\begin{abstract}
Purpose: To evaluate the association between rotator cuff tear (RCT) size and long head biceps tendon (LHBT) pathology. Methods: We retrospectively enrolled 202 consecutive patients (114 women and 88 men with mean age at surgery of 62.14 years [SD, 7.73]) who underwent arthroscopic rotator cuff repair for different sized full-thickness RCTs. LHBT pathology was evaluated considering the presence of inflammation, section alteration, loss of integrity, dislocation, dynamic instability, and absence. The site of LHBT pathology was evaluated considering 3 portions: (1) the insertional element; (2) the free intra-articular portion; (3) the part that enters the intertubercular groove. Statistics were evluated. Results: The LHBT was absent in 22 cases $(10.9 \%): 2,4,15$, and 1 patients with small, large, massive, and subscapularis RCTs, respectively. A significant correlation was found between the prevalence of LHBT absence and massive RCTs $(P<.001)$. In 53 patients $(26 \%)$, there was a healthy LHBT; a healthy LHBT was present in $47 \%, 20 \%$ and $8 \%$ of small, large and massive RCTs, respectively. A significant correlation between LHBT inflammation, section alteration, loss of integrity, and RCT severity was found $(P<.001, P<.001$, and $)$. The insertional portion was the most involved $(57 \%$ of cases); RCT severity was significantly associated with the number of involved portions $(P<.001)$. Conclusions: Shoulder LHBT pathology is associated with increasing rotator cuff tear size. Clinical Relevance: Surgeons should be aware that biceps pathology is particularly prevalent in patients with larger RTCs.
\end{abstract}

I nflammation, morphological alteration and loss of integrity are long head biceps tendon (LHBT) conditions commonly associated with rotator cuff tears (RCTs). Literature has reported that LHBT integrity is compromised in $36.1 \%^{1}$ to $88 \%^{2}$ of patients who undergo RCT repair. An increasing incidence of LHBT pathology with RCT size has also been hypothesized. ${ }^{1,3-6}$ Unfortunately, reports in the literature have

From the Department of Anatomical, Histological, Forensic Medicine and Orthopaedics Sciences, Sapienza University of Rome, Rome Italy (V.C., M.R., S.G.); Istituto Clinico Ortopedico Traumatologico (ICOT), Latina, Italy (V.C., M.R., S.G.); Sapienza Univerisity, Sant'Andrea Hospital, Rome, Italy (J.P.S.); and Shoulder Unit, San Feliciano Clinic, Rome, Italy (S.C.).

The authors report no conflicts of interest or sources of funding. Full ICMJE author disclosure forms are available for this article online, as supplementary material.

Received January 16, 2021; accepted July 18, 2021.

Address correspondence to Vittorio Candela, M.D., Department of Anatomical, Histological, Forensic Medicine and Orthopaedics Sciences, Sapienza University of Rome; Istituto Clinico Ortopedico Traumatologico (ICOT), Latina, Italy, Piazzale Aldo Moro 5, 00185, Rome, Italy. E-mail: vittorio.candela@yahoo.it

(C) 2021 THE AUTHORS. Published by Elsevier Inc. on behalf of the Arthroscopy Association of North America. This is an open access article under the CC BY-NC-ND license (http://creativecommons.org/licenses/by-nc-nd/4.0/).

2666-061X/2165

https://doi.org/10.1016/j.asmr.2021.07.013 given little importance to the pathoanatomy of the LHBT, instead focusing mainly on LHBT treatments ${ }^{7-11}$ and on the clinical outcomes of such procedures. A few authors ${ }^{12-18}$ have tried to describe the possible lesions of the LHBT that are associated with common shoulder injuries; however, the information is unclear due to the contemporary consideration of both intra-articular and subacromial pathologies. Chen et al. ${ }^{4,5}$ evaluated the prevalence of LHBT pathology in a homogeneous sample of patients with complete RCTs. However, information is limited only to gross types of LHBT pathologies, with few details about severity; furthermore, no data regarding the involved area are present. The purpose of this study was to evaluate the association between RCT size and LHBT pathology. Our hypothesis was that RCT size is closely related to both type and location of LHBT pathology.

\section{Methods}

The present study is a retrospective analysis of consecutive patients who underwent arthroscopic repair of a full-thickness RCT between January 2018 and September 2020. Diagnosis of RCT was made by a physical examination and by radiographic and magnetic resonance imaging. 
Table 1. The Long Head Biceps Tendon Pathology Classification in Patients With Rotator Cuff Tear

\begin{tabular}{|c|c|}
\hline Type & Location \\
\hline Inflammation & A: insertional \\
\hline 0 : no inflammation & B: free intra-articular portion \\
\hline $\begin{array}{l}\text { 1: low to medium-grade severity } \\
\text { (characterized by a light red } \\
\text { color) }\end{array}$ & $\begin{array}{c}\text { C: the portion that enters the } \\
\text { intertubercular groove }\end{array}$ \\
\hline $\begin{array}{l}\text { 2: high-grade severity } \\
\text { (characterized by a heavy red } \\
\text { color) }\end{array}$ & \\
\hline Section & \\
\hline 0: normal section & \\
\hline 1: flattened tendon & \\
\hline Integrity & \\
\hline 0: normal tendon & \\
\hline $\begin{array}{l}\text { 1: low-grade delamination (> } \\
50 \% \text { of residual tendon } \\
\text { thickness) }\end{array}$ & \\
\hline $\begin{array}{l}\text { 2: high-grade delamination } \\
\text { ( }<50 \% \text { of residual tendon } \\
\text { thickness })\end{array}$ & \\
\hline
\end{tabular}

Exclusion criteria for all participants were primary moderate or severe osteoarthritis of the operated or contralateral shoulder, traumatic cause of RCT, a previous operation on the shoulder, a history of shoulder dislocations, concomitant or previous frozen shoulder, or inflammatory joint disease.

One of the authors (SG) performed all arthroscopic treatments, with patients in the beach-chair position under general anesthesia and interscalene block. RCT size was confirmed intraoperatively according to the Southern California Orthopedic Institute classification $^{19}$; a small RCT tear was classified as Type I; large and massive RCTs were classified as Types II and III, respectively. Isolated subscapularis RCTs were considered Type IV.

All surgeries were recorded (Stryker IT Recording System, Formello RM, Italy). LHBT dislocation, dynamic instability and absence were registered. LHBT pathology was evaluated considering 3 degrees of tendon inflammation, 2 degrees of section alteration, and 3 degrees of tendon integrity. Furthermore, 3 portions were considered. The adopted LHBT pathology classification is summarized in Table 1 . The video review was performed in every patient by 3 different physicians so as to assess interobserver reliability.

According to the laws of our country, this study did not need ethics committee approval.

\section{Statistical Analysis}

A Shapiro-Wilk test was used to assess the normal data distribution. Categorical variables were calculated using frequencies and proportions, and continuous data were estimated using standard deviations and ranges.

An Independent $t$ test was used to analyze differences between the 2 groups. Also, differences among 3 or more groups for all data were analyzed using a l-way ANOVA test. Significant levels of multiple comparisons were adjusted using the Bonferroni procedure. The $\chi^{2}$ test was conducted for statistical analysis concerning categorical data. Calculated $P$ values were 2 -sided, a $P$ value of less than 0.05 was considered significant, and the range of confidence interval (CI) was 95\%, when appropriate. Statistical analysis was performed using the Statistical Package for Social Sciences version 25 (SPSS, IBM, Armonk, NY, USA).

\section{Results}

The final study group was composed of 202 patients with different sized RCTs because 25 patients were excluded (4 severe osteoarthritis of the operated shoulder; 6 traumatic causes of RCT; 7 previous operations on the shoulder, 4 histories of shoulder dislocations, 4 inflammatory joint disease). The baseline characteristics of the studied group are reported in Table 2 . The mean time between the onset of symptoms and surgery was 5 months (range, 2-12 months).

Intraclass correlation coefficient (ICC) results showed good to excellent reliability for all the parameters: degree of inflammation (ICC 0.95; ICC 95\% 0.89-0.99); section alteration (ICC 0.90; ICC 95\% 0.85-0.95); and tendon integrity (ICC 0.91; ICC 95\% 0.86-0.95).

During arthroscopic evaluation, the LHBT was absent in 22 cases $(10.9 \%)$. According to the tear dimension, LHBT absence was found in 2, 4, 15, and 1 patients with small, large, massive, and subscapularis RCTs. A significant correlation was found between the prevalence of LHBT absence and massive RCTs $(P<.001)$. In 2 patients, an LHBT dynamic instability was found; in both cases, a Type IV RCT was diagnosed. In 6 patients, a dislocation of the LHBT was present: Type II and IV RCTs were present in 4 and 2 cases, respectively. In the remaining 174 patients, signs of LHBT pathology were present; 53 patients $(26 \%)$ had healthy LHBTs, with no signs of inflammation or instability and no morphological alterations. Considering RCT severity, a healthy LHBT was present in $47 \%, 20 \%$ and $8 \%$ of small, large and massive RCTs, respectively.

The prevalence of absent, mild and severe LHBT inflammation is shown in Fig 1. A significant

Table 2. Baseline Characteristics of the Studied Group

\begin{tabular}{lcrr}
\hline & Number $(\%)$ & Mean age (SD) & Range \\
\hline Total & $202(100 \%)$ & $61.14(7.74)$ & $40-75$ \\
$\quad$ Female & $114(56.4 \%)$ & $60.0(8.77)$ & $46-75$ \\
$\quad$ Male & $88(43.6 \%)$ & $61.78(7.07)$ & $40-75$ \\
Rotator cuff tear & & & \\
$\quad$ Type I & $76(37.6 \%)$ & $58.26(7.81)$ & $40-75$ \\
Type II & $66(32.7 \%)$ & $61.68(6.57)$ & $49-74$ \\
Type III & $48(23.8 \%)$ & $64.17(7.90)$ & $46-75$ \\
Type IV & $12(5.9 \%)$ & $64.33(6,89)$ & $57-75$ \\
\hline
\end{tabular}




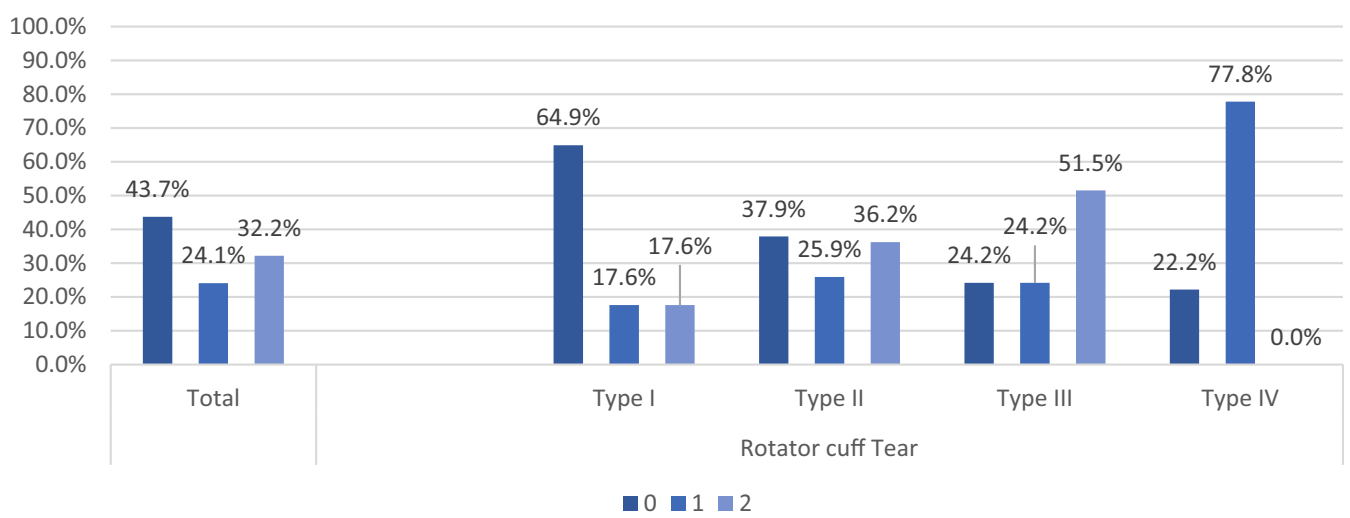

Fig 1. Prevalence of inflammation of the long head biceps tendon according to rotator cuff tear severity and according to the long head biceps tendon pathology classification (0, absence; 1, low grade; 2, high grade).

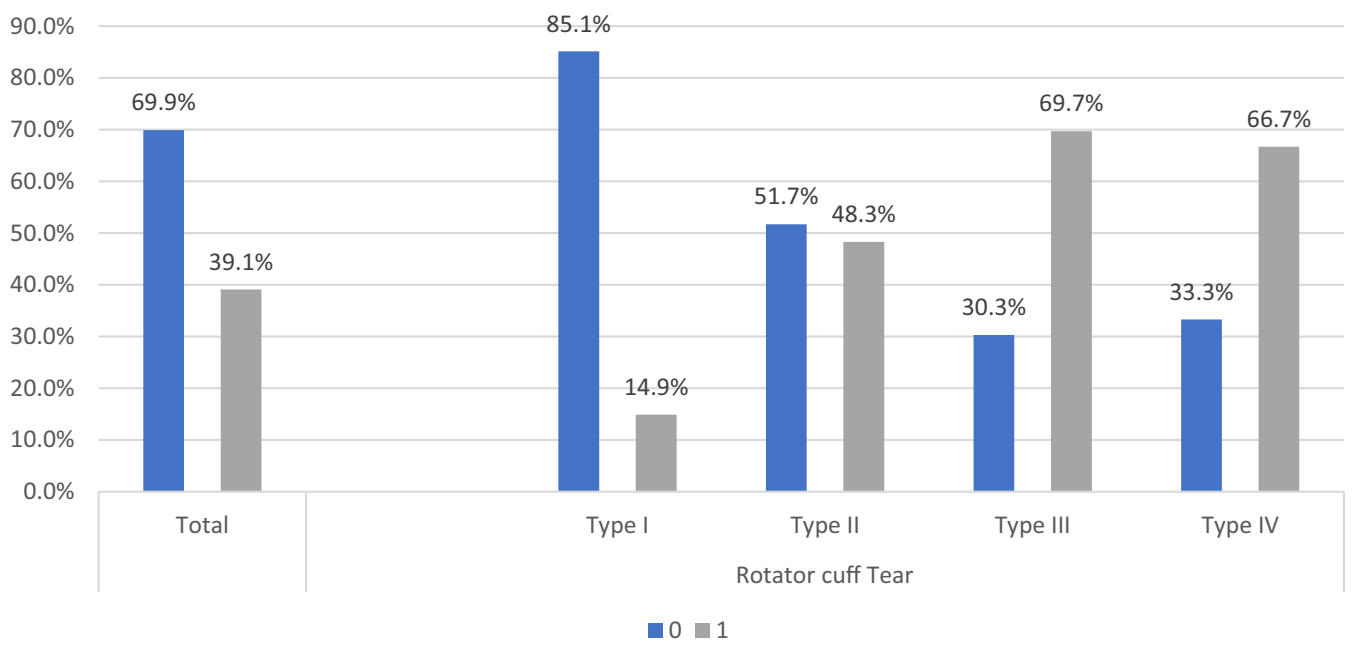

Fig 2. Prevalence of section alteration of the long head biceps tendon according to rotator cuff tear severity and according to the long head biceps tendon pathology classification (0, normal tendon; 1, flattened tendon).

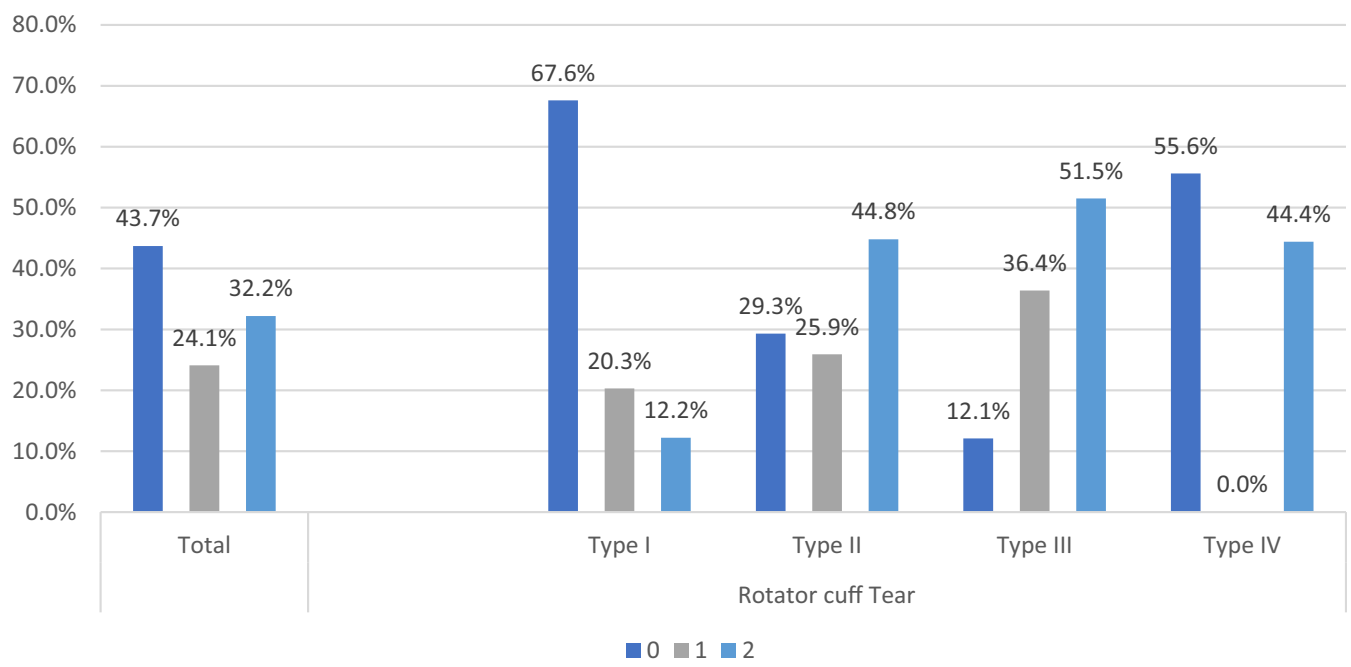

Fig 3. Prevalence of integrity alteration of the long head biceps tendon according to rotator cuff tear severity and according to the long head biceps tendon pathology classification $(0$, normal tendon; 1, low grade delamination [ $>50 \%$ of residual tendon thickness]; 2, high-grade delamination $(<50 \%$ of residual tendon thickness). 


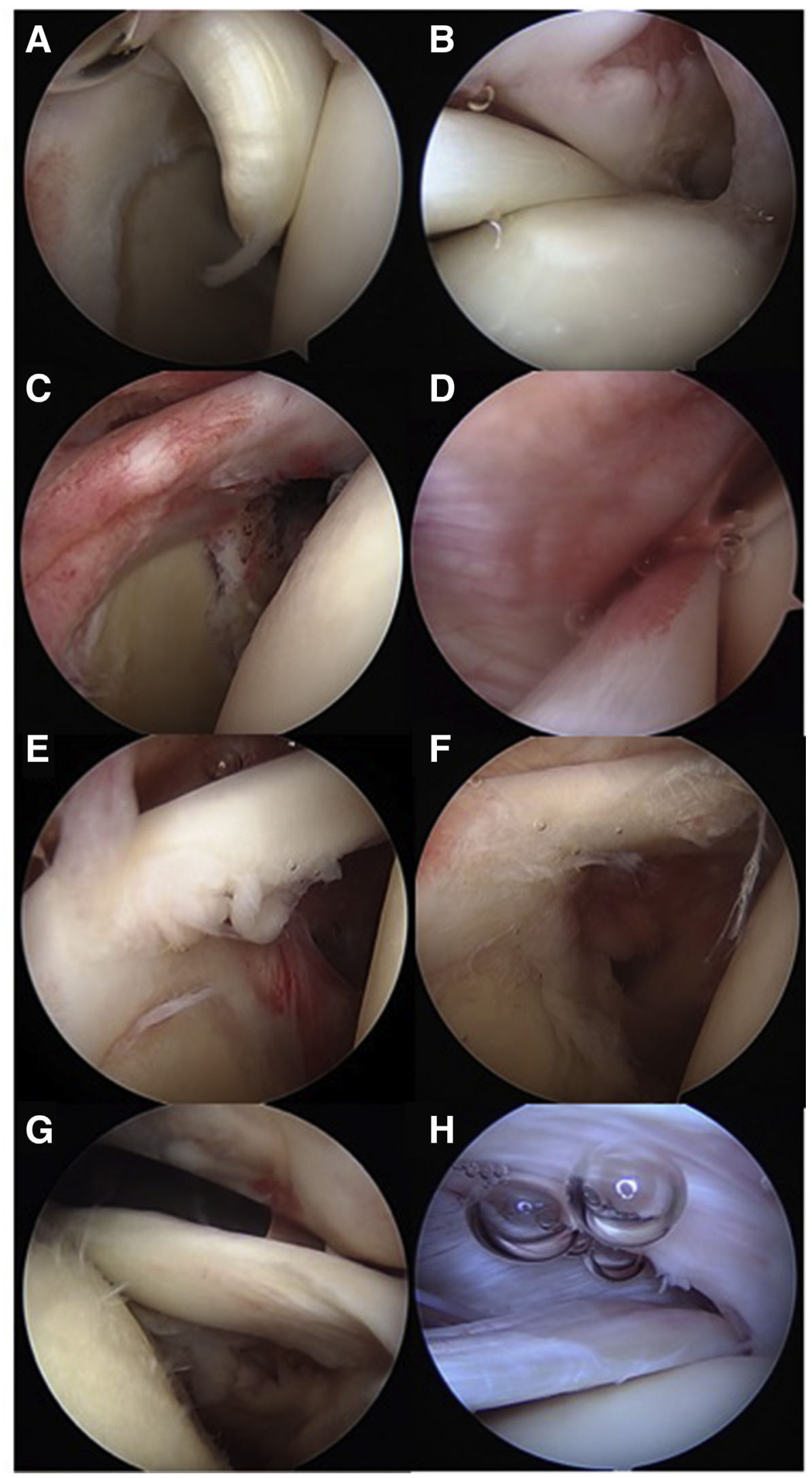

Fig 4. Long head biceps tendon (LHBT) pathology. Arthroscopic view from the posterior portal: (A) Absence of the tendon; only a small insertional portion is present. (B) Dislocation of the LHBT associated with a subscapularis tear. (C) High-grade inflammation of the insertional portion (portion A) of the LHBT. (D) Low-grade inflammation of the distal portion (portion C) of the LHBT. (E) Low-grade delamination ( $>50 \%$ of residual tendon thickness) of portion $\mathrm{A}$ of the LHBT. (F) High-grade delamination $(>50 \%$ of residual tendon thickness) of portions A and B of the LHBT. (G) Flattened LHBT in the portion A. (H) Flattened LHBT in portion C. 


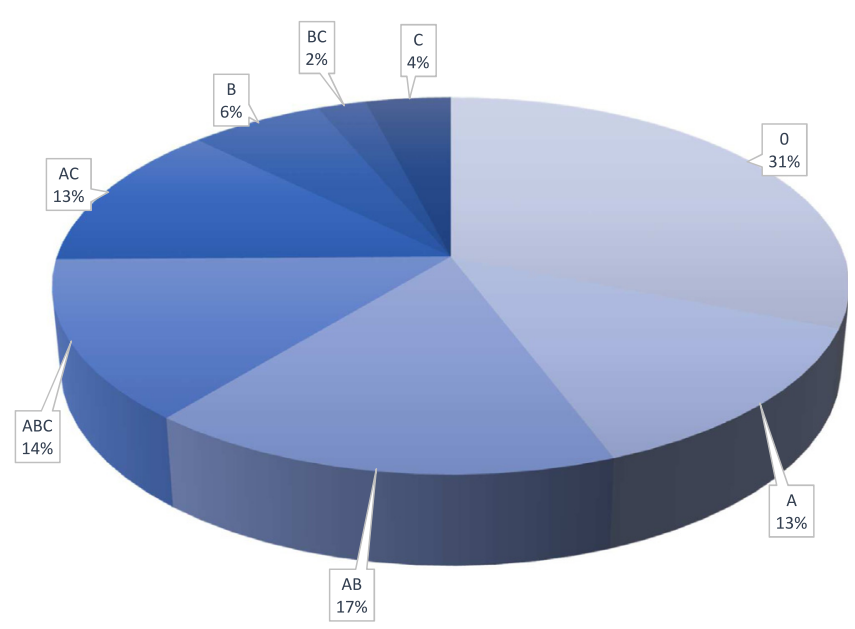

Fig 5. Distribution of LHBT pathology into the 3investigated portions. (A) Insertional; (B) free intra-articular; (C) intertubercular groove portion.

correlation between RCT severity and increasing LHBT inflammation was found $(P<.001)$.

Data according to LHBT section are reported in Fig 2. The prevalence of a flattened LHBT was significantly associated with RCT severity $(P<.001)$. Fig 3 shows the prevalence of alteration in LHBT integrity in the studied group. A significantly higher prevalence of no section alteration of the LHBT was found in patients with small RCTs; on the other hand, type II and III RCTs were significantly associated with the presence of $<50 \%$ of residual LHBT thickness $(P<.001)$ (Fig 4$)$.

Fig 5 shows the distribution of LHBT degeneration according to the examined portions. The distribution of LHBT degeneration in the 3 portions, according to tear severity, is shown in Table 3. A significant correlation was found $(P<.001)$.

\section{Discussion}

The present study demonstrates that the prevalence of LHBT pathology in the setting of RCTs is high (74\%) and is significantly correlated with RCT severity. The prevalence of a healthy LHBT was nearly $50 \%$ in patients with small RCTs but decreased up to $8 \%$ in massive RCTs. The insertional portion of the LHBT was affected in more than half of the cases $(57 \%)$, and the vast majority of patients $(46 \%)$ had at least 2 affected portions; the number of involved areas correlates with RCT severity.

The present study considered 3 degrees of inflammation and structural integrity and 2 degrees of morphological alteration, together with the presence of LHBT dislocation, instability and absence. Furthermore, the site of LHBT pathology was also described in detail, considering 3 portions: insertional, intra-articular and the portion that enters the intertubercular groove within 2 extra-articular centimeters.

In our cohort, 53 patients $(26 \%)$ had healthy LHBTs. The prevalence we found is superior to that of Chen et al., ${ }^{5}$ who found a rate of healthy LHBTs of $18 \%$. However, Chen et al.'s sample included traumatic RCTs, which are associated with a greater prevalence of LHBT inflammation and SLAP tears. ${ }^{20}$

Considering RCT dimension, a healthy LHBT was present in $47 \%, 20 \%$ and $8 \%$ of small, large and massive RCTs, respectively. The role of the LHBT as a pain generator could be redefined by this finding; patients with small RCTs suffer the highest pain intensity $^{21,22}$; however, in half of these patients, the LHBT was healthy. Considering the progression of LHBT pathology with RCT severity, our study stresses, again, the importance of early treatment of small RCTs to preserve the LHBT from degeneration.

In our sample, only 2 cases of dynamic instability of the LHBT were found. Walch et al. ${ }^{18}$ reported 2 types of subluxation: over the superior part and on the medial rim. In our series, the upper third of the subscapularis tendon was torn in both cases and, consequently, the subluxation was medial.

Dislocation of the LHBT has been regarded as being associated with large or massive RCTs or an evolution of subluxation. ${ }^{23}$ Four types of dislocation have been described: inside subscapularis, intra-articular dislocation with subscapularis tear, intra-articular dislocation with subscapularis-transverse ligament tear, and extraarticular dislocation. In our series, 6 patients with LHBT dislocations were found; in 4 cases, a large RCT was associated with an extra-articular dislocation with an empty groove; in the remaining patients, a subscapularis tear and a consequent intra-articular dislocation were diagnosed.

Considering the $74 \%$ of patients with signs of LHBT pathology, our study documented a significant association between LHBT inflammation, morphological alteration and loss of integrity; RCT severity demonstrated how RCTs affect glenohumeral biomechanics and LHBT anatomy, starting from a small lesion. The intra-articular portion of the LHBT is exposed to shear, compressive and tension loading, ${ }^{5}$ whereas the extraarticular portion is subjected primarily to tensional strain. ${ }^{24,25}$ More than 30 years ago, Neviaser et al. ${ }^{26}$ hypothesized a correlation between the severity of rotator cuff disease and the extent of inflammatory changes in the LHBT. Refior ${ }^{27}$ and Phlafer $^{28}$ proposed a model of repetitive traction, friction and glenohumeral rotation, possibly due to upward migration of the humeral head in patients with dysfunctional rotator cuffs. Pressure and shear forces can occur on the tendon at distinct, anatomically narrow sites, resulting in such degenerative changes as fibrosis, thickening, collagen disorganization, scar tissue, and rupture. ${ }^{29}$ The prevalence of LHBT absence confirmed the progression of pathology with the increasing RCT severity; in $31 \%$ of patients with massive RCTs, the LHBT was not present during the intra-articular evaluation. 


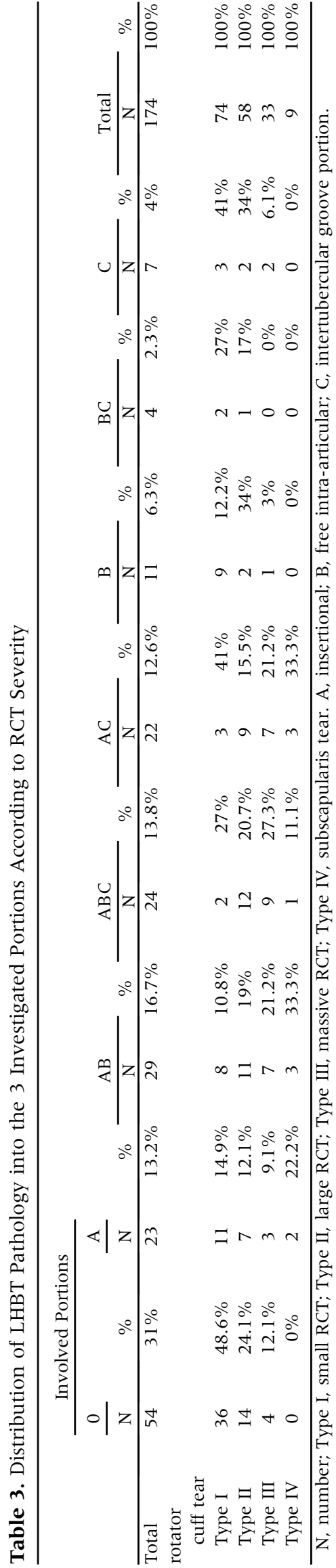

Considering the site of LHBT pathology, previous studies ${ }^{4,5,30}$ have pointed out that the proximal portion of the LHBT is more prone to pathology because that area is subject to significant hypovascularity and to increased mechanical strain with the progression of RCTs. ${ }^{31,32}$ Our study confirmed that the insertional portion is the most involved; surprisingly, it is not the only area affected by pathology. The vast majority of patients with LHBT pathology had involvement of 2 portions and, as for the degree of inflammation, morphological alteration and loss of integrity, RCT severity was found to be correlated with the number of involved portions.

The prevalence of LHBT absence in the studied group confirmed that pathology increases with RCT severity; in fact, in $31 \%$ of patients with massive RCTs, the LHBT was not present. These data could help surgeons who plan a superior capsular reconstruction with biceps tendon to plan alternative strategies.

\section{Limitations}

This study has limitations that should be cited. RCT severity also includes tear shape, retraction, fatty degeneration, and quality of rotator cuff tissue, all of which were not reported. However, tear dimension classification was strictly applied. No subclassification of subscapularis biceps pulley lesions was performed to analyze their influence on LHB pathologies.

\section{Conclusion}

Pathology of the shoulder LHBT is associated with increasing size of an RCT.

\section{References}

1. Desai SS, Mata HK. Long head of biceps tendon pathology and results of tenotomy in full-thickness reparable rotator cuff tear. Arthroscopy 2017;33:1971-1976.

2. Kim YS, Jeong JY, Lee HJ. Arthroscopic tenodesis of the long head of the biceps tendon. JBJS Essent Surg Tech 2017;7:e19.

3. Kim Y-K, Kim D-W, Lee J-H. Long head of the biceps tendon lesion associated with rotator cuff tear. J Korean Shoulder Elbow Soc 2010;13:64-71.

4. Chen CH, Hsu KY, Chen WJ, Shih CH. Incidence and severity of biceps long head tendon lesion in patients with complete rotator cuff tears. J Trauma 2005;58:1189-1193.

5. Chen CH, Su CI, Wang KC, et al. Classification and analysis of pathology of the long head of the biceps tendon in complete rotator cuff tears. Chang Gung Med J 2012;35:263-270.

6. Wu PT, Jou IM, Yang CC, et al. The severity of the long head biceps tendinopathy in patients with chronic rotator cuff tears: Macroscopic versus microscopic results. J Shoulder Elbow Surg 2014;23:1099-1106.

7. Mardani-Kivi M, Keyhani S, Ebrahim-Zadeh MH, Hashemi-Motlagh K, Saheb-Ekhtiari K. Rotator cuff tear with concomitant long head of biceps tendon (LHBT) degeneration: What is the preferred choice? Open 
subpectoral versus arthroscopic intraarticular tenodesis. J Orthop Traumatol 2019;20:26.

8. Belay ES, Wittstein JR, Garrigues GE, et al. Biceps tenotomy has earlier pain relief compared to biceps tenodesis: A randomized prospective study. Knee Surg Sports Traumatol Arthrosc 2019;27:4032-4037.

9. MacDonald P, Verhulst F, McRae S, et al. Biceps tenodesis versus tenotomy in the treatment of lesions of the long head of the biceps tendon in patients undergoing arthroscopic shoulder surgery: A prospective doubleblinded randomized controlled trial. Am J Sports Med 2020;48:1439-1449.

10. Castricini R, Familiari F, De Gori M, et al. Tenodesis is not superior to tenotomy in the treatment of the long head of biceps tendon lesions. Knee Surg Sports Traumatol Arthrose 2018;26:169-175.

11. Castagna A, Borroni M, Garofalo R, et al. Deep partial rotator cuff tear: Transtendon repair or tear completion and repair? A randomized clinical trial. Knee Surg Sports Traumatol Arthrosc 2015;23:460-463.

12. Slätis $P$, Aalto K. Medial dislocation of the tendon of the long head of the biceps brachii. Acta Orthop Scand 1979;50:73-77.

13. Hedtmann H, Fett G, Heers G. Läsionen im bereich des rotatore intervalls und der langen bizepssehne. In: Gohlke F, Hedtmann A, eds. Schulter: das Standardwerk für klinik und praxis. Stuttgart: Georg Thieme Verlag, 2002;310-316.

14. Yamaguchi K, Bindra R. Disorders of the biceps tendon. In: Iannotti J, Williams GR, eds. Disorders of the shoulder: diagnosis and management. Philadelphia, PA: Lippincott Williams and Wilkins, 1999;159-190.

15. Habermeyer P, Magosch P, Pritsch M, Scheibel MT, Lichtenberg S. Anterosuperior impingement of the shoulder as a result of pulley lesions: A prospective arthroscopic study. J Shoulder Elbow Surg 2004;13:5-12.

16. Murthi AM, Vosburgh CL, Neviaser TJ. The incidence of pathologic changes of the long head of the biceps tendon. J Shoulder Elbow Surg 2000;9:382-385.

17. Lafosse L, Reiland Y, Baier GP, Toussaint B, Jost B. Anterior and posterior instability of the long head of the biceps tendon in rotator cuff tears: A new classification based on arthroscopic observations. Arthroscopy 2007;23:73-80.

18. Walch G, Nové-Josserand L, Boileau P, Levigne C. Subluxations and dislocations of the tendon of the long head of the biceps. J Shoulder Elb Surg 1998;7:A1.

19. Snyder SJ. Arthroscopic classification of rotator cuff lesions and surgical decision making, Shoulder Arthroscopy. 2nd ed. Philadelphia: JB Lippincott, 2002;204. ISBN: 0781735017, 9780781735018.

20. Miller C, Savoie FH. Glenohumeral abnormalities associated with full-thickness tears of the rotator cuff. Orthopaedic Rev 1994;23:159-162.

21. Gumina S, Di Giorgio G, Bertino A, Della Rocca C, Sardella B, Postacchini F. Inflammatory infiltrate of the edges of a torn rotator cuff. Int Orthop 2006;30: 371-374.

22. Gumina S, Candela V, Passaretti D, et al. Intensity and distribution of shoulder pain in patients with different sized postero-superior rotator cuff tears. J Shoulder Elbow Surg 2014;23:807-813.

23. Cofield RH, Parvizi J, Hoffmeyer PJ, Lanzer WL, Ilstrup DM, Rowland CM. Surgical repair of chronic rotator cuff tears: A prospective long-term study. J Bone Joint Surg Am 2001;83:71-77.

24. Ditsios K, Agathangelidis F, Boutsiadis A, Karataglis D, Papadopoulos P. Long head of the biceps pathology combined with rotator cuff tears. Adv Orthop 2012;2012:405472.

25. Gleason PD, Beall DP, Sanders TG, et al. The transverse humeral ligament: A separate anatomical structure or a continuation of the osseous attachment of the rotator cuff? Am J Sports Med 2006;34:72-77.

26. Neviaser TJ, Neviaser RJ, Neviaser JS. The four-in-one arthroplasty for the painful arc syndrome. Clin Orthop Relat Res 1982;163:107-112.

27. Refior HJ, Sowa D. Long tendon of the biceps brachii: Sites of predilection for degenerative lesions. J Shoulder Elbow Surg 1995;4:436-440.

28. Pfahler M, Branner S, Refior HJ. The role of the bicipital groove in tendopathy of the long biceps tendon. J Shoulder Elbow Surg 1999;8:419-424. PMID: 10543593.

29. Nho SJ, Strauss EJ, Lenart BA, et al. Long head of the biceps tendinopathy: Diagnosis and management. J Am Acad Orthop Surg 2010;18:645-656.

30. Ahmad CS, DiSipio C, Lester J, Gardner TR, Levine WN, Bigliani LU. Factors affecting dropped biceps deformity after tenotomy of the long head of the biceps tendon. Arthroscopy 2007;23:537-541. PMID: 17478286.

31. Boileau P, Ahrens PM, Hatzidakis AM. Entrapment of the long head of the biceps tendon: the hourglass biceps: A cause of pain and locking of the shoulder. J Shoulder Elbow Surg 2004; 13:249-257.

32. Post M, Benca P. Primary tendinitis of the long head of the biceps. Clin Orthop Relat Res 1989;246:117-125. 